\title{
Respiratory Rate as a Physiological Response to Dental Anxiety
}

\author{
Cahyanti Wydiastuti Susilo ${ }^{1}$, Eva Fauziah ${ }^{2 *}$ \\ ${ }^{1}$ Pediatric Dentistry Residency Program, Faculty of Dentistry, Universitas Indonesia, Jakarta \\ 10430, Indonesia. \\ ${ }^{2}$ Pediatric Dentistry Department, Faculty of Dentistry, Universitas Indonesia, Jakarta 10430, Indo- \\ nesia. \\ *Email: eva_dens@yahoo.com
}

\begin{abstract}
Dental treatment is often associated with pain, fear, and anxiety. Unfortunately, children with dental anxiety tend to resist dental treatment and behave uncooperatively during dental procedures. Dental treatment is known to be stressful and is often associated with a physiological response; therefore, dental anxiety can lead to physiological changes in the body, including increases in the respiratory rate, heart rate, and blood pressure. The psychophysiological response due to dental anxiety is related to an increase in autonomic nervous system activity. Anxiety is a basic emotion resulting from individual's defense mechanisms; therefore, the respiratory changes in an anxiety response are controlled by the brain center that is related to emotion. Respiration is regulated mainly in the brainstem, but complex interactions between the brainstem, limbic system, and cortical structure of the brain affect the final output of respiration. The amygdala, part of the limbic system, is responsible for processing negative emotions; therefore, measuring physiological responses, such as the respiratory rate, plays an important role in behavioral assessment. This paper presents the respiratory rate as one of the physiological responses to dental anxiety.
\end{abstract}

Keywords: Dental anxiety, Physiological response, Respiratory rate

\section{Introduction}

Dental anxiety is a challenge for a practitioner trying to provide appropriate dental treatment. A lack of communication and skill in managing children with anxiety can lead to misdiagnosis and suboptimal treatment. A dentist must know the predisposing factors and etiology and be able to provide an individualized approach for patients with dental anxiety. If the anxiety is not diagnosed, the patient will avoid treatment and develop complications such as oral problems, poor aesthetics, and social problems [1].

A study conducted by Soares et al. in 2014 showed that the prevalence of dental anxiety was $46 \%$ in 100 Brazilian children aged 5-8 years [2]. A high level of anxiety will result in uncooperative children or adults, wasted time, and less effective dental treatment. Children manifest dental anxiety in different ways. Some respond with interruptive behavior, while others respond via physiological reactions such as an increased respiratory rate, rapid heartbeat, sweating, and high blood pressure [1, 3]. 
Many studies have shown a correlation between anxiety and breathing patterns $[4,5,6]$. For example, Masaoka et al. reported that an unpleasant emotion could affect the respiratory pattern, and there was a positive correlation between the anxiety score and respiratory rate [4]. Their next study showed that the respiratory frequency can increase in the absence of changes in the metabolic rate in subjects exhibiting high levels of anxiety during stressful tasks [5]. Another study reported that anxiety is one of the factors that can increase the respiratory rate in individuals with idiopathic hyperventilation [6].

Respiration is primarily regulated in the brainstem, which controls the body's metabolism and homeostasis. However, respiration can change as a response to a negative emotion, such as panic, anxiety, and pain, or to positive emotions, such as happiness, romance, and the feeling of relief $[7,8]$. The amygdala, part of the limbic system, is associated with anxiety and an increased respiratory rate, particularly in subjects with high anxiety [6]. The amygdala evaluates external stimuli and determines whether a stimulus is safe or dangerous. If a stimulus is dangerous, the amygdala simultaneously responds to the fear and anxiety with physiological changes for self-protection [9]. Therefore, measurement of the physiological response could be a basis for determining the level of emotion in certain situations. This is an important aid to dentists in determining an appropriate approach for optimal dental treatment.

\section{Dental Anxiety}

The term "anxiety" comes from the German word "angst" and was first introduced to psychology by Freud in 1936 [10]. Anxiety describes the brain's response to a harmful situation, which becomes a stimulus for someone to actively avoid something. This response is a basic emotion starting in infancy and continuing throughout childhood [11. The response to danger or a harmful situation is called the fight or flight response, and when in danger, an individual will attempt to flee or fight to survive. Moreover, anxiety can be a reaction to a stressful situation or something dangerous. The manifestation in an anxious individual comes in the form of a physiological response such as dyspnea, palpitations, and fear of the reoccurrence of the incident [12]. They will tend to avoid the threat in their surroundings.

Dental anxiety is oftentimes correlated with anxiety, pain, and fear, and the prevalence of anxiety in these patients varies worldwide. In North America, the population with dental anxiety ranges from $10 \%$ to $20 \%$, while it is $11 \%$ in Germany and $10 \%$ in Norway [1]. Soares et al. reported that $46 \%$ of 100 Brazilian children aged 5-8 years had dental anxiety [2]. The main causes of dental anxiety are pain and fear of pain; therefore, an anxious patient is more sensitive to pain. In addition, other factors such as a previous traumatic dental experience and a fear of needles or blood can contribute to dental anxiety $[13,14]$. A fear of the dentist consists of dental anxiety, dental fear, and dental phobia; dental anxiety is generally benign, whereas dental phobia is the most intensive type. There are strong correlations between dental anxiety and poor oral health, an increased level of caries, and behavioral management problems in children, because an anxious patient tends to resist dental treatment, and this usually leads to irregular dental visits [1]. 
Subjective or objective methods can be used to assess dental anxiety. A subjective method can consist of a questionnaire or visual scale, with the most commonly used visual scales being the Venham Picture Test and Facial Image Scale. The objective method is an alternative to the subjective measurement, and the measurement of the physiological response plays an important role in behavioral assessment. Psychophysiological responses due to dental anxiety are related to an increase in autonomic nervous system activity. These responses can include increased blood pressure and a rapid heartbeat in the cardiovascular system, breathlessness in the respiratory system, constipation in the digestive system, and increased sweat production in the integumentary system [3].

\section{Respiration}

Respiration plays a vital role in an individual's life, and the respiratory system is responsible for maintaining the balance of oxygen, carbon dioxide, and hydrogen in the body. Excesses of carbon dioxide and hydrogen stimulate the respiratory system, resulting in increased inspiration and expiration signals in the respiratory muscles [15].

The respiratory system is controlled mainly in the brainstem, and there are two neural mechanisms that regulate respiration: voluntary control and automatic control [16]. Voluntary control occurs in the cerebral cortex and thalamus, which send impulses via the corticospinal tracts to the respiratory motor neurons. They both coordinate respiration with voluntary motor activity that involves the lungs and chest wall, like singing, coughing, sneezing, defecation, and responses to anxiety and fear. However, automatic control is driven by a group of pacemaker cells in the medulla oblongata. The impulses are received by the pacemaker cells, which activate the motor neurons in the cervical and thoracic spinal cord that innervate the inspiratory muscles. Motor neurons in the expiratory muscles are inhibited while the inspiratory muscles are active and vice versa. Automatic or metabolic breathing is the basic system, and during this kind of breathing, both the respiratory rate and depth of breathing (tidal volume) are regulated so that the partial pressures of the arterial oxygen and carbon dioxide are equal. Normal breathing in a resting state is automatic and uses little energy [16, 17].

The breathing mechanism is comprised of inspiratory and expiratory processes. Inspiration is an active process initiated by neural impulses from the respiratory control centers in the medulla and pons. Neural impulses stimulate inspiratory muscles to contract, causing the thoracic cavity to expand and lower the pressure in the pleural space around the lungs. As the pressure decreases, the lungs will passively expand, causing the pressure in the alveoli to fall and air to flow into the alveoli until the pressure is equal to that of the atmospheric pressure in the airway. Conversely, expiration is a passive process, which occurs via the elastic recoil of the lungs as they return to their original positions. The alveolar and pleural pressures rise, and the gas flows out of the lung [17]. 


\section{Correlation between Respiration and Dental Anxiety}

Dental treatment is stressful and often associated with a physiological response in a child [18]. West et al. reported that the injection of the local anesthetic evoked the greatest physiological response, including increased skin conductance and pulse rate, to dental procedures [18]. Negative emotions, such as anxiety, can cause physiological changes in the human body. These physiological changes are controlled by the autonomic nervous system, which regulates the involuntary functions of the body, including breathing control, blood pressure, heart rate, and sexual function [19]. Activation of the sympathetic nerve branch of the autonomic nervous system is dominant during emergency conditions, which recruit the fight or flight response, and during sports. This branch is activated by positive or negative emotions, such as happiness, anxiety, and anger [20]. One study conducted by O'Brian et al. revealed that the autonomic nervous system in subjects with dental anxiety can be activated by medicinal odors like that of eugenol [21]. Moreover, Sanadhya et al. reported that the pulse rate, blood pressure, and electrocardiographic (ECG) changes are increased significantly in children with high anxiety levels during tooth extraction procedures [22].

Respiration is regulated in the brainstem for both metabolic and homeostatic purposes. Yet, the respiration rate can change in response to emotional changes, including both negative emotions (panic, anxiety, and pain) and positive emotions (happiness, romance, and relief) $[7,8]$. Anxiety is the basic emotion of an individual's defense mechanism; therefore, respiratory changes in the response to anxiety are controlled by the brain center that is related to emotion. Dental anxiety is very common in childhood. It is the main reason for avoiding the dentist and can provoke a psychophysiological response before or during dental treatment. A change in the respiratory rate is one type of psychophysiological response. Eitner et al. reported a positive correlation between the respiratory rate and dental anxiety, and they found that the respiratory rate in a patient with dental anxiety was increased before the dental treatment began [23].

Complex interactions between the brainstem, limbic system, and cortical structure of the brain can affect the final respiratory output. The pole in the temporal lobe and amygdala in the limbic system are related to anxiety and an increase in respiratory frequency [6]. In addition, some studies have reported that the amygdala plays an important role in processing negative emotions such as fear and anxiety $[8,9]$. The amygdala is one of the components of the midbrain that evaluates an external stimulus and determines whether the stimulus is safe or harmful. If the stimulus is harmful, the amygdala will simultaneously compensate for the fear and anxiety with physiological changes for self-protection. Therefore, measurement of the physiological response can be used to determine the level of emotion [9].

\section{Measurement Devices For The Respiratory Rate}

The respiratory rate can be obtained by observation, palpation, or auscultation of the chest while counting inhalations per minute. Normal breathing reflects a rela- 
tively constant respiratory rate and volume, and this breathing is automatic and effortless. The character and rate of respiration is influenced by pathological conditions; therefore, it is important to know the normal respiration frequency standards. The normal respiratory rate is $20-28$ breaths/minute in preschool children aged $3-$ 5 years and $20-30$ breaths/minute in children aged 6-8 years [24]. Tachypnea and bradypnea are two respiratory abnormalities. Tachypnea is characterized by an increase in the respiratory rate due to a variety of causes. In healthy children, tachypnea can occur due to excitement or fear, yet an obstruction in the airway or infection can also cause tachypnea. Conversely, a decrease in the respiratory rate is a known characteristic of bradypnea. Central respiratory depression can cause a slow respiratory rate, with sedatives, alkalosis, and increased intracranial pressure being common causes [24].

The medical devices used to measure the respiratory rate are divided into contact and noncontact methods. One disadvantage of the contact method devices is that the child may refuse to wear the device, potentially causing stress and altering the respiratory rate. Therefore, this method is not suitable for younger children or acute conditions. Contact method devices make direct contact with the patient's body, measuring the chest and abdominal movements and oxygen saturation and calculating the respiratory rate from an ECG [25].

With non-contact-based respiratory rate monitoring, the device does not contact the patient's body. It is suitable for younger children, such as newborn babies, and in acute conditions. These devices include infrared thermography, video data, radar systems, and mobile applications. Infrared thermography is used to monitor the fluctuations in the facial skin temperature during exhalation, which increase the temperature of the tip of the nose [25]. The video data method records the chest movements of the patient, whereas the radar system detects chest movements during respiration with an electromagnetic wave. The respiration frequency can be calculated by using the pulse-Doppler principle, which has been in use since 1970 [26]. This type of device can be used to monitor periodic movements of the body during breathing at a certain distance. The advantages of the radar system are that it does not need an electrode or belt that contacts the patient's body, it can be used over a long distance, and wireless transfer of the respiratory rate data to a central unit is possible. The latest portable device for determining the respiratory rate is via a mobile application using a built-in camera on a mobile device; however, this device has not been clinically tested and validated [25].

\section{Conclusion}

Children with dental anxiety tend to resist dental treatment and behave uncooperatively during a dental procedure. A dentist should be able to identify an anxious patient and provide special management to ensure effective and efficient dental treatment. One type of anxiety measurement is via psychophysiological responses, such as the respiratory rate, which change due to an increase in autonomic nervous system activity. 


\section{References}

1. Jovanović-Medojević M, Nešković J, Medojević A. Dental Anxiety: Etiology and Treatment Options. Serbian Dent J. 2015;62(4):174-83.

2. Soares FC, Lima RA, de Barros MV, Colares V. Factors associated with dental anxiety in Brazilian children of 5 to 8 years. Pesqui Bras Odontopediatria Clin Integr. 2014;14(2):97-105.

3. Jimeno FG, Bielsa SY, Fernández CC, Rodríguez AL, Bellido MM. Objective and subjective measures for assessing anxiety in paediatric dental patients. Eur J Paediatr Dent. 2011; 12:239-44.

4. Masaoka Y, Homma I. Anxiety and respiratory patterns: their relationship during mental stress and physical load. Int J Psychophysiol. 1997;27(2):153-9.

5. Masaoka Y, Homma I. The effect of anticipatory anxiety on breathing and metabolism in humans. Respir Physiol. 2001;128(2):171-7.

6. Masaoka Y, Jack S, Warburton CJ, Homma I. Breathing patterns associated with trait anxiety and breathlessness in humans. Jpn J Physiol. 2004;54(5):465-70.

7. Holstege G, Beers CM, Subramanian HH. The Central Nervous System Control of Respiration. Elsevier; 2014 Apr 17.

8. Homma I, Masaoka Y. Breathing rhythms and emotions. Exp Physiol. 2008 Sep 1;93(9):1011-21.

9. Masaoka Y, Homma I. Amygdala and emotional breathing in humans. In Post-genomic perspectives in modeling and control of breathing 2004 (pp. 9-14). Springer, Boston, MA.

10. Freud S. The problem of anxiety. Read Books Ltd; 2013 Apr 16.

11. Beesdo K, Knappe S, Pine DS. Anxiety and anxiety disorders in children and adolescents: developmental issues and implications for DSM-V. Psych Clin. 2009;32(3):483524 .

12. Craske MG, Stein MB. Anxiety. J. Anxiety. Lancet. 2016;6736(16):1-12.

13. Nigam AG, Marwah N, Goenka P, Chaudhry A. Correlation of general anxiety and dental anxiety in children aged 3 to 5 years: A clinical survey. J Int Oral Health. 2013;5(6):18.

14. Porritt J, Buchanan H, Hall M, Gilchrist F, Marshman Z. Assessing children's dental anxiety: a systematic review of current measures. Community Dent Oral Epidemiol 2013;41(2):130-42.

15. Hall JE, Guyton AC. Guyton and Hall Medical Physiology Text Book. 9th ed. (Setiawan I, ed.). Philadelphia: W.B Saunders Company; 1997.

16. Barrett KE, Barman SM, Boitano S, Brooks H. Ganong's Review of Medical Physiology, 24th Edition. 24th ed. Singapore: McGraw-Hill Education; 2012.

17. Levy MN, Berne RM. Principles of Physiology. Third. Missouri: Elsevier Mosby; 2000.

18. West GA, Reid KH, Bastawi AE. Autonomic responses to dental procedures in pedodontic patients during a standard restoration session. J Dent Res. 1983;62(6):728-32.

19. Kreibig SD. Autonomic nervous system activity in emotion: A review. Biol. Psychol. 2010;84(3):394-421.

20. Shiota MN, Neufeld SL, Yeung WH, Moser SE, Perea EF. Feeling good: autonomic nervous system responding in five positive emotions. Emotion. 2011;11(6):1368.

21. Robin O, Alaoui-Ismaili O, Dittmar A, Vernet-Maury E. Emotional responses evoked by dental odors: an evaluation from autonomic parameters. J Dent Res. 1998; $77(8): 1638-46$. 
22. Sanadhya YK, Sanadhya S, Jalihal S, Nagarajappa R, Ramesh G, Tak M. Hemodynamic, ventilator, and ECG changes in pediatric patients undergoing extractionJ Indian Soc Pedod Prev Dent. 2013;31(1):10.

23. Eitner S, SCHULTZE - MOSGAU S, Heckmann J, Wichmann M, Holst S. Changes in neurophysiologic parameters in a patient with dental anxiety by hypnosis during surgical treatment. J Oral Rehabil. 2006;33(7):496-500.

24. Poole AE, Macko DJ. Pediatric vital signs: recording methods and interpretations. Pediatr Dent. 1984;6(1):10-6.

25. Daw W, Kingshott R, Saatchi R, Burke D, Holloway A, Travis J, Evans R, Jones A, Hughes B, Elphick H. Medical Devices for Measuring Respiratory Rate in Children. J Adv Bio Eng Technol. 2016; 3:21-7.

26. Droitcour AD, Seto TB, Park BK, Yamada S, Vergara A, El Hourani C, Shing T, Yuen A, Lubecke VM, Boric-Lubecke O. Non-contact respiratory rate measurement validation for hospitalized patients. InEngineering in Medicine and Biology Society, 2009. EMBC 2009. Annual International Conference of the IEEE 2009 Sep 3 (pp. 4812-4815). IEEE. 